\title{
Estimating the driver function of a cardiovascular system model
}

\author{
Stevenson, D.J. * Hann, C.E. ${ }^{*}$ Chase, J.G. ${ }^{*}$ Revie, J. * \\ Shaw, G.M. ${ }^{* *}$ Desaive, T. ${ }^{* * *}$ Lambermont, B. ${ }^{* * *}$ \\ Ghuysen, A. ${ }^{* * *}$ Kolh, P. ${ }^{* * *}$ Heldmann, S. ${ }^{* * *}$ \\ * Department of Mechanical Engineering, University of Canterbury, \\ Christchurch, New Zealand \\ ** Department of Intensive Care, Christchurch Hospital, Christchurch, \\ New Zealand \\ *** Hemodynamics Research Laboratory, University of Liege, Belgium \\ **** Department of Mechanical Engineering, TU Darmstadt, Germany
}

\begin{abstract}
The time varying elastance of the left and right ventricles, also called the driver functions, are the inputs to a previously developed cardiovascular model. However these functions cannot normally be measured directly in an Intensive Care Unit (ICU), as they require both ventricle volume and pressure waveforms. This paper develops a method to construct both driver functions from measurements which are more commonly available in an ICU. Specifically the aortic pressure and pulmonary artery pressure waveforms. The information used from the pulmonary artery pressure can be found from the central venous pressure and ECG, however these were not available for this study. This paper also details a method to locate specific points of interest in the aortic and pulmonary artery pressures, such as the dicrotic notch. The driver functions that were constructed with a median error of $1.32 \%$ and $2.54 \%$ for the left and right respectively, with a 90 percentile of $3.67 \%$ and $9.43 \%$ respectively, and tracked all the physiologically meaningful points on the driver function, such as the start and end of ejection and the maximum ascending gradient.
\end{abstract}

\section{INTRODUCTION}

Left and right pressure-volume (PV) loops contain a large amount of information on cardiac function including all the states of filling, contraction, ejection and relaxation, see Guyton and Hall (2000). The formula:

$$
e l(t)=\frac{P_{l v}(t)}{V_{l v}(t)-V_{d}}
$$

where

$$
\begin{aligned}
V_{d} & \equiv \text { deadspace } \\
P_{l v}(t) & \equiv \text { left ventricle pressure } \\
V_{l v}(t) & \equiv \text { left ventricle volume }
\end{aligned}
$$

is commonly called time varying elastance, see Oommen et al. (2003), and provides a load-independent measure of heart function that can represent cardiac muscle activation. The time varying elastance is typically normalised to one, and the resulting shape can be used as the input or driver function into lumped parameter models of the cardiovascular system, see Chung et al. (1997). The shape of the driver function thus approximates the change in cardiac muscle activation over time, and implicitly contains information on the ventricle pressure and volume profiles. However, left and right ventricle pressures and volumes are not typically measured, and in usual intensive care practice are never known. Yet, the concept of PV loops is critical to understanding, for example the effect of inotropes on cardiac function.
This research aims to estimate the left and right ventricle driver functions from available measurements in the Intensive Care Unit (ICU). The driver function then provides the input into a previously developed 8 chamber cardiovascular system (CVS) model, see Starfinger et al. (2008). The overall goal is to use this CVS model to transform standard ICU data into a more physiologically clear picture. ICU data typically includes, central venous pressure, ECG, arterial pressure, $\mathrm{SPO}_{2}$, cardiac output and global end-diastolic volume (GEDV). Note that the latter two measurements come from PiCCO, see PULSION (2010). Thus there is a wide range of diverse data available but complex interactions and the body's natural reflex responses can make accurate diagnosis very difficult. A validated computer model can provide a patients specific representation to assist diagnosis and optimise therapy.

The data investigated in the paper is from a porcine model of pulmonary embolism, which contains significant changes in hemodynamics and is thus a solid case study to demonstrate the driver function estimation methods. These methods will provide the foundation for extending to human data in upcoming clinical trials at the Christchurch ICU.

\section{METHODOLOGY}

The general shape of the driver function has four main sections: an exponential rise (A), a shoulder section (B) and two exponential decays $(\mathrm{C}$ and $\mathrm{D})$ as shown in Figure 1. Note that the deadspace is always assumed 
to be 0 since it has little effect on the overall shape as demonstrated in Figure 2.

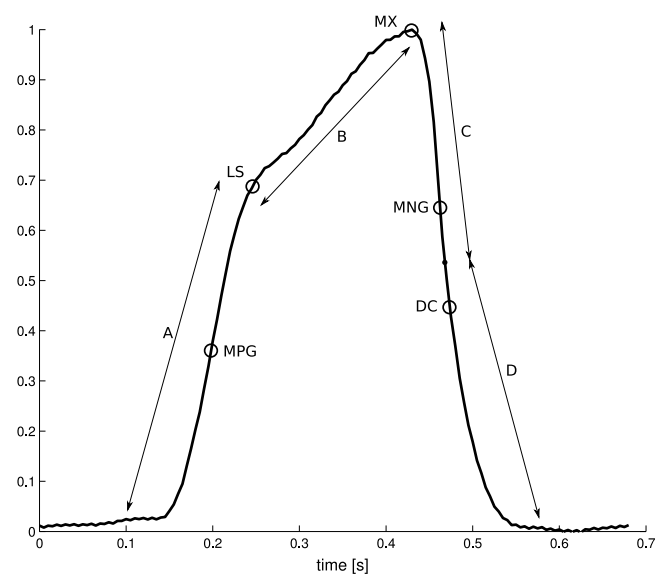

Fig. 1. An example of a typical driver function for the left side of the heart

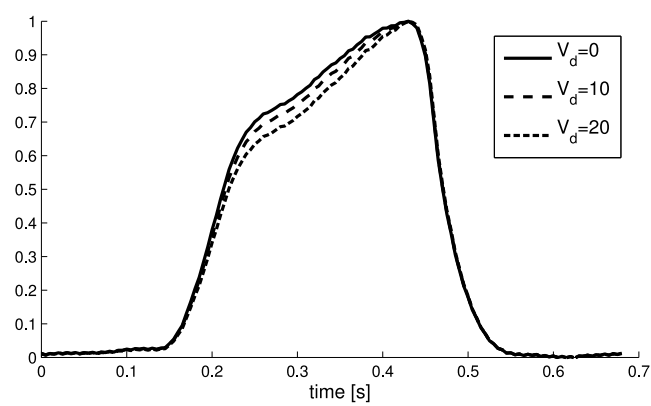

Fig. 2. Three driver functions with different deadspace

Each of the sections have a physiological interpretation, as well the time intervals in between. The key features include:

- the time between the two inflection points (ascending and descending) is the period of ejection

- the peak of the driver function corresponds to the re-polarization of the ventricle

- the shoulder section is a slower portion of ejection that relates to the twisting of the ventricle.

The exact shape of the driver function requires measurement of both the ventricle volume and pressure, which is not available in an ICU. However the aortic pressure waveform, which is measured in the ICU, contains a significant amount of information that can be used to reconstruct the left driver function. To avoid the requirement of an extra pulmonary artery catheter, the right driver function is estimated based only on timing in the pulmonary artery pressure which could be obtained from other less invasive measurements of central venous pressure and ECG.

For describing the methods to follow a naming convention is given.

$$
\begin{aligned}
e l & \equiv \text { left driver function } \\
e r & \equiv \text { right driver function } \\
P_{a o} & \equiv \text { aortic pressure } \\
P_{p a} & \equiv \text { pulmonary artery pressure }
\end{aligned}
$$$$
D M P G \equiv \text { driver maximum postive gradient }
$$$$
M N \equiv \text { minimum point }
$$$$
M P G \equiv \text { maximum positive gradient }
$$$$
L S \equiv \text { left shoulder }
$$

$M X \equiv$ maximum point

$R S \equiv$ right shouder

$M N G \equiv$ maximum negative gradient

$D N \equiv$ dicrotic notch

$D C \equiv$ dicrotic crest

$t_{a, b} \equiv$ time of point $b$ on waveform $a$

The error of the reconstruction is defined by the median percentage and 90\% confidence interval, of the distance between the real and reconstructed waveforms. The distance is defined as the shortest distance between the real and reconstructed waveform at any given point on the real waveform, after both waveforms had been normalised to the heart beat (producing a waveform confined in a $1 \mathrm{x} 1$ box). This distance is defined by:

$$
d=\sqrt{\left(t_{e l, a}-t_{e l_{a p r x}, b}\right)^{2}+\left(e l_{a}-e l_{a p r x, b}\right)^{2}}
$$

where, $b$ is found for every $a$ such that $d$ (at every $a$ ) is minimised.

\subsection{Left driver function}

The points of interest ( $M P G, L S, M N G, D C$ ) can be correlated to specific features in the aortic pressure, note that $t_{e l, M X} \equiv c_{2}$ from Equation (17). These correlations are defined:

$$
\begin{aligned}
& t_{e l, M N G}=A_{t, M N G} \cdot t_{P_{a o}, D N}+B_{t, M N G} \\
& e l_{M N G}=A_{M N G} \cdot t_{P_{a o}, M N G}+B_{M N G} \\
& e l_{M N G}^{\prime}=A_{M N G^{\prime}} \cdot \log _{e}\left(\frac{P_{a o, M N G}}{\text { period }}\right)+B_{M N G^{\prime}} \\
& t_{e l, M P G}=A_{t, M P G} \cdot t_{P_{a o}, D M P G}+B_{t, M P G} \\
& e l_{M P G}=A_{M P G} \cdot \frac{P_{a o, M P G}^{\prime}}{\text { period }}+B_{M P G} \\
& e l_{M P G}^{\prime}=A_{M P G^{\prime}} \cdot \frac{P_{a o, M P G}^{\prime}}{\text { period }}+B_{M P G^{\prime}} \\
& t_{e l, L S}=A_{t, L S} \cdot t_{P_{a o}, M P G}+B_{t, L S} \\
& e l_{L S}=A_{L S} \cdot \frac{P_{a o, L S}}{\text { period } \cdot P_{a o, M X}}+B_{L S} \\
& t_{e l, D C} \equiv t_{P_{a o}, D C} \\
& e l_{D C}=A_{D C} \cdot \frac{P_{a o, D C}^{\prime}}{\text { period }}+B_{D C}
\end{aligned}
$$

where, for example, $t_{P_{a o}, D N}$ is the time of the dicrotic notch in the aortic pressure waveform, as was defined in Equation (3).

A continuous estimation of the driver function is defined: 


$$
\begin{aligned}
& e l_{\text {apr } x}(t)= F_{1}(t), \quad 0<t \leq t_{e l, L S} \\
&= F_{1}\left(t_{b}\right)+ \\
&\left(\frac{F_{2}\left(t_{e l, M X}\right)-F_{1}\left(t_{e l, L S}\right)}{t_{e l, M X}-t_{e l, L S}}\right) \cdot\left(t-t_{e l, M X}\right), \\
& \quad t_{e l, L S} \leq t \leq t_{e l, M X} \\
&= F_{2}(t), \quad t_{e l, M X} \leq t \leq t_{d} \\
&= F_{3}(t), \quad t_{d} \leq t \leq \text { period } \\
& t_{d}=\frac{t_{e l, M N G}+t_{e l, D C}}{2}
\end{aligned}
$$

where

$$
F_{i}=a_{i} \cdot e^{-b_{i}\left(t-c_{i}\right)^{2}}, \quad i=1,2,3
$$

and $a_{i}, b_{i}$, and $c_{i}$, are unknowns. For the ascending portion $(i=1)$ these unknowns are determined from: the inflection point $e l_{M P G}$, the maximum ascending gradient $e l_{M P G}^{\prime}$, and the shoulder height of the driver function, $e l_{L S}$. This shoulder point is assumed to the peak of the function $e l_{\text {aprx }}(t)$ of Equation (16). The physiological justification of this step is that the rise before the shoulder is the initial main contraction of the ventricle. The period after this is twisting of the ventricle which is a different dynamic and therefore should be treated separately. Thus, the essential idea is to fit an exponential wave to the sections A, B, C and D, of Figure 1. The equations to fit these unknowns are defined:

$$
\begin{aligned}
a_{i} & =e_{2} \\
b_{i} & =-\frac{\log \left(e_{1, i} / e_{2, i}\right)}{\left.\exp \left(\log \left(\log \left(e_{1, i} / e_{2, i}\right) \cdot 2 \cdot\left(e_{1, i} / d E_{i}\right)\right) \cdot 2\right)\right)} \\
c_{i} & =-\frac{\log \left(e_{1, i} / e_{2, i}\right) \cdot 2 \cdot e_{1, i}-d E_{i} \cdot t_{1}}{d E_{i}}
\end{aligned}
$$

where

$$
\begin{aligned}
& \left\{e_{1,1}, e_{2,1}, d E_{1}, t_{1,1}\right\} \equiv\left\{e l_{M P G}, e l_{L S}, e l_{M P G}^{\prime}, t_{e l, M P G}\right\} \\
& \left\{e_{1,2}, e_{2,2}, d E_{2}, t_{1,2}\right\} \equiv\left\{e l_{M N G}, 1, e l_{M N G}^{\prime}, t_{e l, M N G}\right\} \\
& \left\{e_{1,3}, e_{2,3}, d E_{3}, t_{1,3}\right\} \equiv\left\{e l_{D C}, 1, e l_{M N G}^{\prime}, t_{P_{a o}, D C}\right\}
\end{aligned}
$$

\subsection{Right driver function}

The points of interest for the right driver function, ( $M P G$, $L S$, and $M N G$ ) are correlated using the aortic pressure waveform and two time points from the pulmonary artery waveform, note that $t_{e r, M X} \equiv c_{2}$ in Equation (17).

The first point $t_{P_{p a}, M N}$ is the beginning of contraction and can be found form the $\mathrm{C}$ wave in the CVP waveform, see Izakovic (2008) and the second points $t_{P_{p a}, D N}$ can be found from the end of ejection or the beginning of the T wave on the ECG, see Guyton and Hall (2000). ECG or CVP waveform data was not available in the study, so a complete validation is left for future work. However, the results certainly prove the concept of estimating the right ventricle driver function with only minimal right side information.

The specific correlations are defined:

$$
\begin{aligned}
t_{e r, M N G} & =A_{t, M N G} \cdot P p a_{t, D N}+B_{t, M N G} \\
t_{e r, M P G} & =A_{t, M P G} \cdot P p a_{t, M N}+B_{t, M P G} \\
e r_{L S} & =A_{L S} \cdot G E D V+B_{L S} \cdot e l_{L S} \\
& +C_{L S} \cdot t_{e l, L S}+D_{L S}
\end{aligned}
$$

$$
\begin{aligned}
e r_{M P G} & =A_{M P G} \cdot G E D V \\
& +B_{M P G} \cdot e l_{M P G}+C_{M P G} \\
e r_{M N G} & =A_{M N G} \cdot G E D V \\
& +B_{M N G} \cdot e l_{M N G}+C_{M N G} \\
e r_{M P G}^{\prime} & =A_{M P G^{\prime}} \cdot G E D V \\
& +B_{M P G^{\prime}} \cdot e l_{M P G}^{\prime}+C_{M P G^{\prime}} \\
e r_{M N G}^{\prime} & =A_{M N G^{\prime}} \cdot G E D V \\
& +B_{M N G^{\prime}} \cdot e l_{M N G}^{\prime}+C_{M N G^{\prime}}
\end{aligned}
$$

The continuous estimation of the right driver function is defined by modifying Equation (15) by replacing $e_{\text {apr }}(t)$ with $e r_{\text {apr } x}(t)$, ignoring $F_{3}(t)$ and defining $t_{d} \equiv$ period. $F_{i}(t)$ is still defined by Equation (17), however the unknowns $a_{i}, b_{i}$, and $c_{i}$ are defined by modifying Equation (18) by replacing el with er for the first two lines, and dispensing with the third.

The only difference between the left and right continuous estimation is that only one exponential decay function is used in the right. Two functions could have been included, however there was very little improvement in the error hence the simpler approach was taken.

\subsection{Processing of the pressure waveforms}

In order for the driver functions to be reconstructed, the aortic pressure and pulmonary artery pressure waveforms need to be processed to accurately and reliably extract the required points that are shown in Figures $3-4$. The method is described only for $P_{a o}$ for simplicity, but the same approach is used for $P_{p a}$.

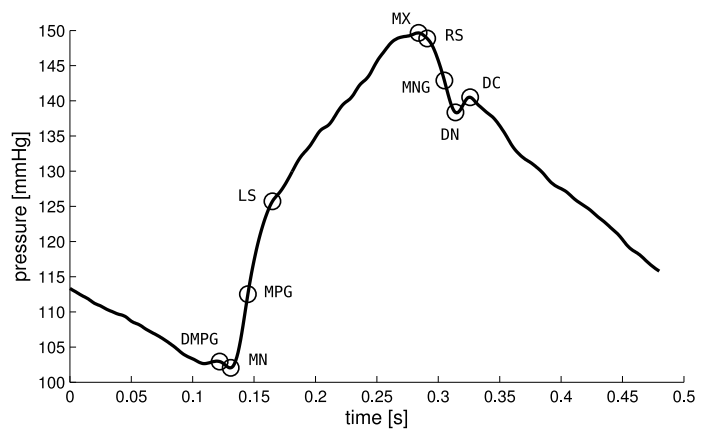

Fig. 3. Aortic pressure over one heart beat, including relevant points defined in Equations (3)

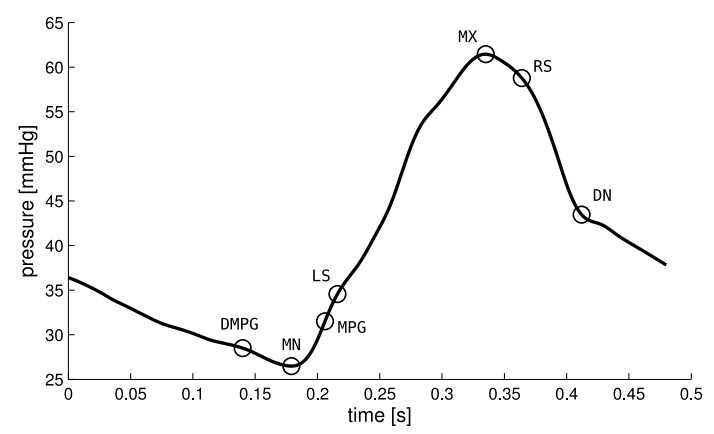

Fig. 4. Pulmonary artery pressure over one heart beat, including relevant points defined in Equations (3) 
The first step is to locate the time of the global maximum of the waveform:

$$
t_{M X} \equiv \text { time of maximum } P_{a o}
$$

To facilitate the extraction of the remaining points, a shear transformation is defined:

$$
\begin{gathered}
(t, X(t)) \rightarrow\left(t, \phi_{\text {shear }}(X(t))\right. \\
\phi_{\text {shear }}(X(t))=X(t)+\text { mt }+c, \quad t_{0}<t<t_{\text {end }}
\end{gathered}
$$

The parameters $t_{0}$ and $t_{\text {end }}$ are set depending on the region of interest. The parameters $m$ and $c$ are chosen such that $\phi_{\text {shear }}\left(X\left(t_{0}\right)\right)=X\left(t_{0}\right)$ and $\phi_{\text {shear }}\left(X\left(t_{\text {end }}\right)\right)=X\left(t_{0}\right)$ which leads to the following equations:

$$
\begin{gathered}
X\left(t_{0}\right)+m t_{0}+c=X\left(t_{0}\right) \\
X\left(t_{\text {end }}\right)+m t_{\text {end }}+c=X\left(t_{0}\right)
\end{gathered}
$$

Solving Equations (28a) and (28b) yields:

$$
\begin{aligned}
m & =\frac{X\left(t_{0}\right)-X\left(t_{\text {end }}\right)}{t_{\text {end }}-t_{0}} \\
c & =\frac{t_{0} X\left(t_{\text {end }}\right)-t_{\text {end }} X\left(t_{0}\right)}{t_{\text {end }}-t_{0}}
\end{aligned}
$$

To better visualize this transformation, imagine putting a line from MN to MX and rotating about MN so that this line is horizontal, but keep all the time points the same. The effect of this transformation is to transform the point LS into a peak of a curve which is easier to locate. Figure 5 shows the result of this transformation. This shear transformation approach is applied to each region of interest.

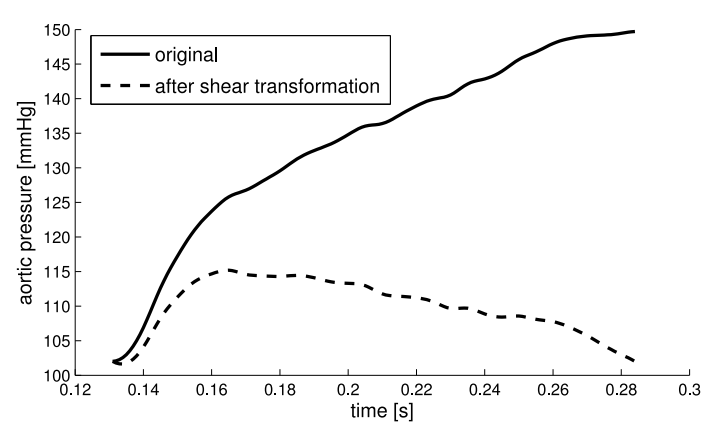

Fig. 5. the section from Figure 3 between $M N$ and $M X$, before and after the shear transformation from Equation (27).

\subsection{1 $M N$ and DMPG:}

The first step is to compute the global minimum of the aortic pressure:

$$
t_{M N a} \equiv \text { time of minimum } P_{a o}
$$

However, $t_{M N a}$ in Equation (30) or alternatively $M N$ in Figure 3 is not always the global minimum of the aortic pressure, as the dip before DMPG can sometimes go lower than MN. To account for this effect, a shear transformation is applied to the section and is defined:

$$
P_{a o, s e c A} \equiv\left\{P_{a o}(t): t_{M N a}<t<t_{M X}\right\}
$$

A second time point is defined:

$$
\begin{aligned}
& \qquad t_{M N a} \equiv \text { time of minimum } \phi_{\text {shear }}\left(P_{a o, \sec A}\right) \\
& \text { where: } \\
& P_{a o, \sec A 2} \equiv\left\{P_{a o}(t): t_{M N a}<t<t_{M X}\right\}
\end{aligned}
$$

which will be the correct minimum if the first trough is smaller than the second, but will be a fake minimum otherwise. In the latter case a point quite far up the waveform is always chosen which make it straight forward to rule out. The method is to define:

$$
\operatorname{error}_{P_{a o}} \equiv \frac{P_{a o}\left(t_{M N b}\right)-P_{a o}\left(t_{M N a}\right)}{P_{a o}\left(t_{M N a}\right)}
$$

and

$$
\begin{array}{rlrl}
t_{M N} & =t_{M N b}, & \text { error }_{P_{a o}}<0.03 \\
& =t_{M N a} & & \text { otherwise }
\end{array}
$$

The constant 0.03 was found empirically, and Equation (35) was found to give good results in all cases.

Looking at Figure 3, it would appear that DMPG would be straightforwardly found by computing the maximum point to the left of MN. However, as Figure 4 shows this case does not always occur, in fact for the pulmonary artery pressure waveform it virtually never occurs. Furthermore, there can be oscillations prior to DMPG that make it more difficult. The procedure to handle this case is to define:

$$
\begin{gathered}
P_{a o, \sec B 1} \equiv\left\{P_{a o}(t): \frac{3}{4} \cdot t_{M N}<t<t_{M N}\right\} \\
t_{A} \equiv \text { time of minimum } \phi_{\text {shear }}\left(P_{a o, \sec B 1}\right) \\
P_{a o, \sec B 2} \equiv\left\{P_{a o}(t): t_{A}<t<t_{M N}\right\} \\
P_{a o, \sec B} \equiv P_{a o, \sec B 1} \text {, if } \exists \text { local max of } \phi_{\text {shear }}\left(P_{a o, \sec B 1}\right) \\
\equiv P_{a o, \sec B 2} \text { otherwise } \\
\text { The time } \\
t_{D M P G} \text { is then found by: } \\
t_{D M P G} \equiv \text { time of maximum } \phi_{\text {shear }}\left(P_{a o, \sec B}\right)
\end{gathered}
$$

\subsection{2 $L S$ and $M P G$ :}

The LS point reveals the point where the slower ejection or twisting of the ventricle occurs. MPG is one of the most important features since it is the commonly called $d P_{a o} d t_{\max }$ value which is well known to be related to ventricle contractility. To locate the LS point a new section is defined:

$$
P_{a o, s e c C} \equiv\left\{P_{a o}(t): t_{M N}<t<t_{M X}\right\}
$$

The simplest approach to finding LS would be to locate the maximum of $\phi_{\text {shear }}\left(P_{a o, s e c C}\right)$ as is clear in Figure 5. However, after the shear transformation a second higher peak can occur as is apparent from looking at Figure 4. The solution is to find both local maxima then have a second criteria to distinguish the correct point. The first maximum is:

$$
t_{L S a} \equiv \text { time of maximum } \phi_{\text {shear }}\left(P_{a o, s e c C}\right)
$$

To account for the case of $t_{L S a}$ begin the wrong point, which means it will be far up the waveform past LS, a section is define:

$$
P_{a o, s e c C 2} \equiv\left\{P_{a o}(t): t_{M N}<t<t_{L S a}\right\}
$$

for this case the correct time of LS should be:

$$
t_{L S b} \equiv \text { time of maximum } \phi_{\text {shear }}\left(P_{a o, s e c C 2}\right)
$$


However in a number of cases $t_{L S a}$ is correct which would mean $t_{L S b}$ is a false point that lies somewhere between MN and LS in Figure 3. To account for both of these cases the solution is:

$$
\begin{aligned}
t_{L S} & =t_{L S a}, \quad\left(t_{L S a}-t_{L S b}\right)<0.02 \\
& =t_{L S b}, \text { otherwise }
\end{aligned}
$$

Where $t_{L S a}$ and $t_{L S b}$ are given by Equations (40) and (42).

The first condition if Equation (43) is due to the curve from MN to LS being close to sigmoidal so that when $t_{L S a}$ is the correct point, $t_{L S b}$ is always very close and within 0.02. In the case where $t_{L S a}$ is incorrect, and thus $t_{L S b}$ is the correct point, $t_{L S a}-t_{L S b}$ is much greater than 0.02 , in fact is always between 0.05 and 0.1 . Hence Equation (43) is robust.

Once $t_{L S}$ is found the time point corresponding to MPG in Figure 3 is defined:

$$
t_{M P G} \equiv \text { time of maximum }\left\{\frac{d}{d t}\left(P_{a o}(t)\right): t_{M N}<t<t_{L S}\right\}
$$

\subsubsection{DN, RS, DC and summary:}

The dicrotic notch (DN) corresponds to the end of ejection. The time point $t_{D N}$ is defined:

$$
t_{D N} \equiv \text { time of minimum } \phi_{\text {shear }}\left(P_{a o, s e c D}\right)
$$

where:

$$
\begin{gathered}
P_{a o, s e c D} \equiv\left\{P_{a o}(t): t_{M X 2}<t<t_{\text {end }}\right\} \\
t_{M X 2} \equiv \text { time of maximum }\left\{P_{a o}(t): t_{D N a}<t<t_{\text {end }}\right\}
\end{gathered}
$$

$$
t_{D N a} \equiv t_{M N}+\frac{\text { period }}{5}
$$

The time point $t_{D N a}$ in Equation (48) thus limits the values that the $t_{D N}$ can take.

Note that the simplest way of defining $t_{D N}$ would be to use $t_{M X}$ from Equation (26) in place of $t_{M X 2}$ in Equation (46). However in a number of cases near the end of the trial when the pig is near death the maximum peak of $P_{a o}$ occurs between LS and MX in Figure 3. Physiologically this phenomenon is likely due to the twisting of the ventricle becoming abnormal and dysfunctional. In practice, it is probably too late to save a patient if this occurs, but for completeness this scenario is accounted for.

The value of $t_{R S}$ is defined:

$$
t_{R S} \equiv \text { time of maximum } \phi_{\text {shear }}\left(P_{a o, s e c E}\right)
$$

where:

$$
P_{a o, \sec E} \equiv\left\{P_{a o}(t): t_{M X}<t<t_{D N}\right\}
$$

Finally, and only for $P_{a o}$ the last section is defined:

$$
P_{a o, s e c F} \equiv\left\{P_{a o}(t): t_{D N}<t<t_{D N}+0.03\right\}
$$

and the crest after the dicrotic notch is found by

$$
t_{D C} \equiv \text { time of maximum } P_{a o, s e c F}
$$

A summary of this process follows which also applies to $P_{p a}$ is given as follows:

Step 1. find $t_{M X}$ using Equation (26)

Step 2. find $t_{M N}$ with Equations (30) to (35)

Step 3. find $t_{D M P G}$ with Equations (36) to (38)
Step 4. find $t_{L S}$ with Equations (39) to (43)

Step 5. find $t_{M P G}$ between $M N$ and $L S$, Equation (44)

Step 6. find $t_{D N}$ with Equations (45) to (48)

Step 7. find $t_{R S}$ with Equations (49) to (50)

Step 8. for $P_{a o}$ only, find $t_{D C}$ using Equations (51) to (52)

\section{RESULTS}

\subsection{Correlations}

Most of the correlations show very high $R$ values, as can been seen in Figure 6, especially those relating the time points of the driver functions. Table 1 shows the complete list or correlations for the left and right driver functions. These correlations are based on the data from all five pigs, so are only representative of those used in the reconstructions, see Section 3.2 The column labeled 'errors\%' is the root mean square of the percentage error of the correlation (e.g. percentage difference between the correlations, dashed line in the Figure 6, and the real data).

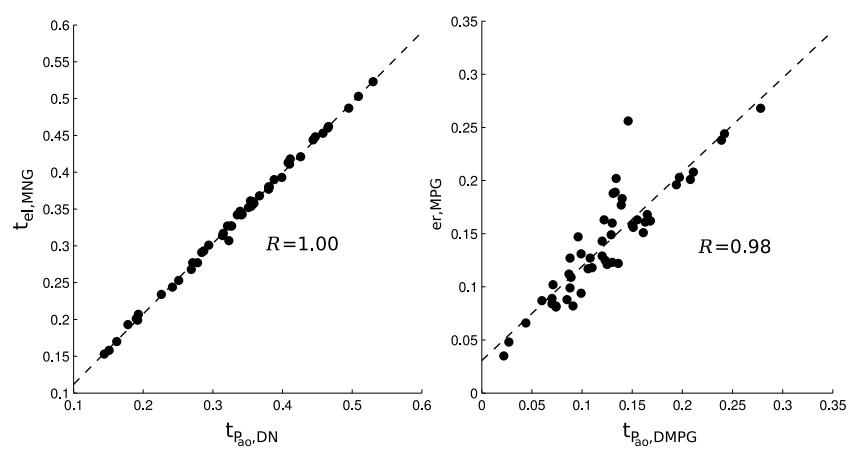

Fig. 6. examples of correlations for the left and rigth ventricle (respectively)

Table 1. Results of the correlations

\begin{tabular}{c|c|c|c} 
Driver point & correlated with & $R$ & Error\% \\
\hline$t_{e l, M N G}$ & $t_{P_{a o}, D N}$ & 1.00 & 1.41 \\
$e l_{M N G}$ & $t_{P_{a o}, M N G}$ & -0.35 & 21.37 \\
$e l_{M N G^{\prime}}$ & $\log _{e}\left(\frac{P_{a o, M N G}^{\prime}}{\text { period }}\right)$ & -0.53 & 18.95 \\
$t_{e l, M P G}$ & $\left.t_{P_{a o}, D M P G}\right)$ & 0.99 & 7.13 \\
$e l_{M P G}$ & $P_{a o, M P G}^{\prime} /$ period & 0.81 & 17.25 \\
$e l_{M P G^{\prime}}$ & $P_{a o, M P G}^{\prime}$ & 0.96 & 14.35 \\
$t_{e l, L S}$ & $t_{P_{a o}, M P G}$ & 1.00 & 2.41 \\
$e l_{L S}$ & $P_{a o, L S}$ & 0.92 & 5.29 \\
$e l_{D C}$ & $P_{a o, D C}^{\prime} / p e r i o d$ & -0.32 & 38.64 \\
\hline \hline$t_{e r, M N G}$ & $t_{P p a, D N}$ & 0.97 & 6.45 \\
$t_{e r, M P G}$ & $t_{P p a, M N}$ & 0.98 & 10.02 \\
$e r_{L S}$ & $G E D V, e l_{L S}, t_{e l, L S}$ & 0.74 & 12.15 \\
$e r_{M P G}$ & $G E D V, l_{M P G}$ & 0.78 & 24.48 \\
$e r_{M N G}^{\prime}$ & $G E D V, l_{M N G}$ & 0.73 & 20.58 \\
$e r_{M P G}^{\prime}$ & $G E D V, e l_{M P G}^{\prime}$ & 0.34 & 17.12 \\
$e r_{M N G}^{\prime}$ & $G E D V, e l_{M N G}^{\prime}$ & 0.42 & 34.05
\end{tabular}

\subsection{Reconstructions}

The reported correlations in Table 1 were reconstructed across all five pigs. To further test the robustness of the approach, when estimating the driver function for a given pig, only the four other pigs were used to develop the correlations. In otherwords, no information from the 
given pig was used. This process is commonly called crossvalidation and is a more realistic test of the method. Figures 7 and 8 show examples from the left and right respectively, that are close to the median percentage error on each side of the heart.

Table 2. Errors in the reconstructed waveforms

\begin{tabular}{r|c|c|} 
& median & 90 percentile \\
\hline left & $1.32 \%$ & $3.67 \%$ \\
\hline right & $2.50 \%$ & $9.43 \%$ \\
\hline
\end{tabular}

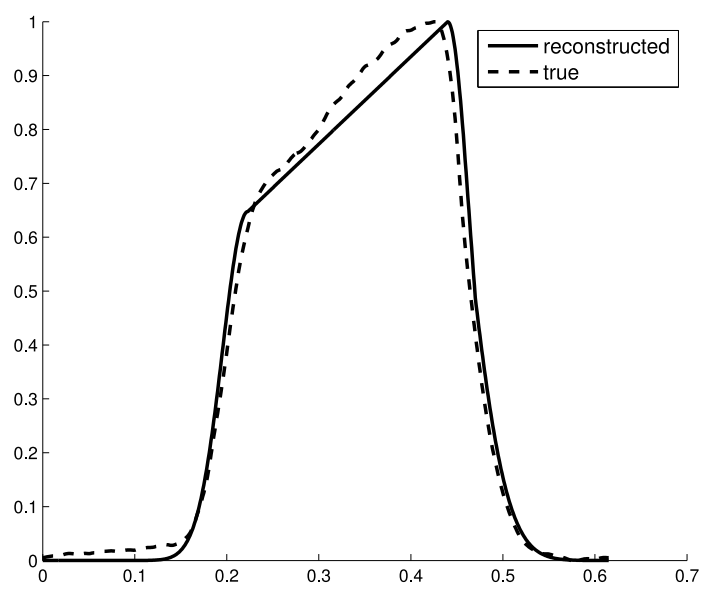

Fig. 7. Left driver function, with an error value of $1.32 \%$

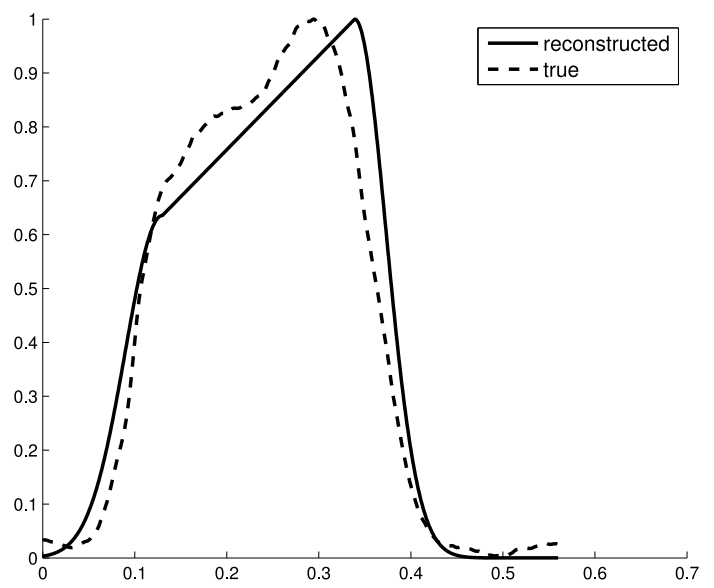

Fig. 8. Right driver function, with an error value of $2.54 \%$

Figure 9 shows four reconstructed driver functions (aloneside the measured driver functions) for the same pig at four different times during the onset of pulmonary embolism. Notice the dramatic decrease in period at $t=255 \mathrm{~min}$, which is captured very well.

\section{DISCUSSION AND CONCLUSION}

A method for identifying patient specific left and right driver functions (time varying elastance of the left and right ventricles), was developed. This method tracks the important time points on the driver functions, such as the start and end of ejection. This was validated on a set of porcine data, which included multiple measurements of five pigs that were induced with pulmonary embolism. A four fold cross-validation was done to predict the driver function.
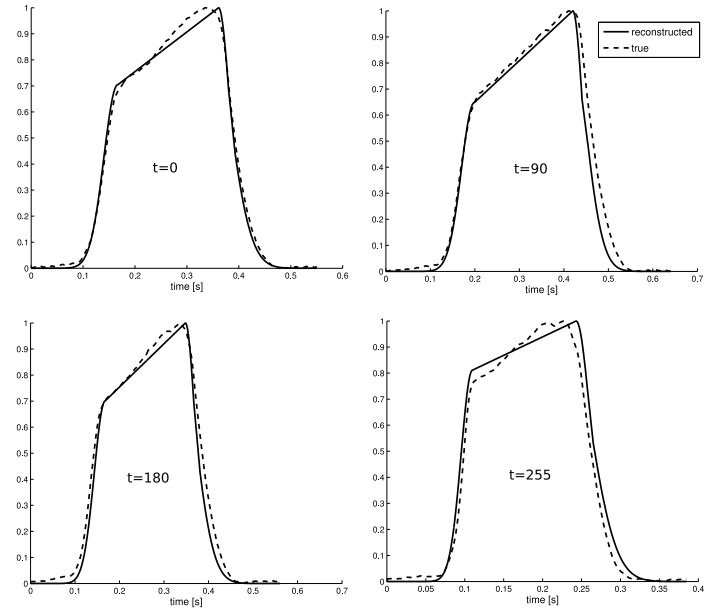

Fig. 9. Four reconstructions of the left driver function, from the same pig at different time intervals (measured in minutes)

This paper also developed a method for extracting specific points from the aortic and pulmonary artery pressure using a novel method of a shear transformation on specific sections of these waveforms. This allowed for complete automation in the process of calculating the correlations and constructing the approximated driver functions. Although this study used the pulmonary artery pressure, this is not specifically needed, as the points found in the pulmonary artery pressure waveform could be located in the central venous pressure and ECG, but the further validation is needed. This means that it is possible to construct the driver functions without any knowledge of the right side of the heart. This is a major advantage for future work in an ICU setting, as pulmonary artery pressure is less commonly measured than aortic pressure.

This paper provides the tools which will lead into future work on humans in an ICU setting, however more validation on human data will be required.

\section{REFERENCES}

Chung, D., Niranjan, S., Clark, J., Bidani, A., and Johnston, W. (1997). A dynamic model of ventricular interaction and pericardial influence. Am. J. Phyisol., 14.

Guyton, A. and Hall, J. (2000). Textbook of Medical Physiology. W. B. Saunders Company, Philadelphia, Tenth edition.

Izakovic, M. (2008). Central venous pressure - evaluation, interpretation, monitoring, clinical implications. Bratisl Lek Listy, 109(4), 185-187.

Oommen, B., Karamanoglu, M., and Kovács, S. (2003). Modeling Time Varying Elastance: The Meangin of "Load-Idependence". Cardiovascular Engineering, 3(4), $123-130$.

PULSION (2010). PULSION Medical Systems. URL http://www.pulsion.com.

Starfinger, C., Chase, J., Hann, C., Shaw, G., Lambermont, B., Ghuysen, A., Kolh, P., Dauby, P., and Desaive, T. (2008). Model-based identification and diagnosis of a porcine model of induced endotoxic shock with hemofiltration. Mathematical Biosciences, 216(2), 132139. 